\title{
COLLECTIVE BEAM INSTABILITIES IN THE TAIWAN LIGHT SOURCE
}

\author{
P.J. Chou', SRRC, Hsinchu, Taiwan \\ A.W. Chao, SLAC, Stanford, CA 94309, USA
}

\begin{abstract}
The storage ring at Taiwan Light Source has experienced a strong collective instability since 1994 . Various cures have been attempted to suppress this instability, including the use of damping antenna, tunable rf plungers, different filling patterns, and rf gap voltage modulation. So far these cures have improved the beam intensity, but the operation remains to be limited by the instability. The dominant phenomenon is the longitudinal coupled bunch instability. The major source of longitudinal impedance is from rf cavities of Doris type. The high-order modes of the cavity were numerically analyzed using a 3-D code GdfidL. The correlation of the observed phenomenon in user operation with high-order modes of rf cavities will be presented. Results of various attempts to suppress beam instabilities will be summarized. Proposed cures for beam instabilities will be discussed.
\end{abstract}

\section{INTRODUCTION}

A longitudinal coupled bunch instability was observed in the storage ring of SRRC prior to 1995[1]. Because the high-order-mode(HOM) damping antenna in the Doristype cavity was not effective to suppress the longitudinal coupled bunch instability at $200 \mathrm{~mA}$ in user operation and because a longitudinal bunch-by-bunch feedback system has not been available, a crash solution was adopted to replace the HOM damping antenna with a tuning plunger in the spring of 1996. The most prominent unstable mode was avoided by shifting the mode frequency with tuning plungers. This patchy solution did not eradicate the longitudinal beam instability completely. User operations still suffered from the beam instability although at a less severe scale. In the fall of 1999 the technique of modulating the $\mathrm{rf}$ gap voltage at nearly twice the synchrotron frequency was implemented[2]. This combined solution was able to maintain a relatively stable operation for most users. Nevertheless, an abrupt orbit distortion occurred in an unpredictable way resulting in unstable photon beam in the user end station. The observation of such abrupt beam instability will be described. Its correlation with the longitudinal HOMs of rf cavities will be presented. Various attempts to cure this beam instability will be described. Results of those attempts will be presented and possible cures will be discussed.

'pjchou@srrc.gov.tw

\section{OBERVATION OF BEAM INSTABILITIES}

\subsection{Closed Orbit vs. Horizontal Beam Size}

vertical difference orbit:

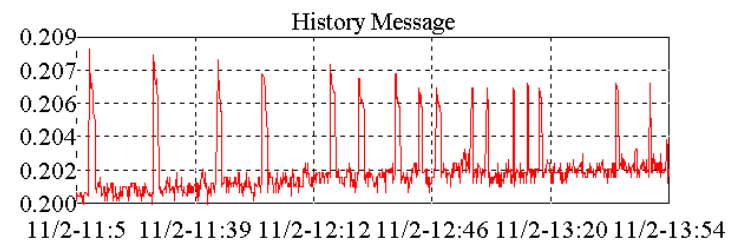

horizontal difference orbit:

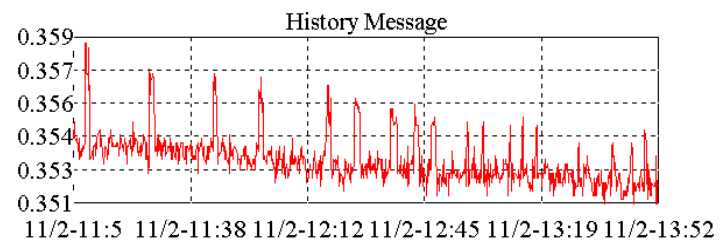

horizontal beam size:

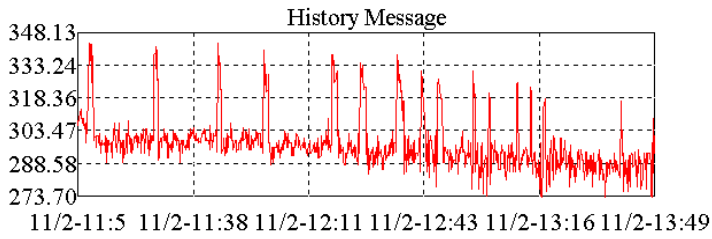

Figure 1: The abrupt distortion of beam orbit and horizontal beam size observed in the same time. The rf voltage modulation was turned on. The vertical scale of beam orbit is in units of $\mathrm{mm}$ and the vertical scale of beam size is in $\mu \mathrm{m}$. The horizontal axis represents the time.

The abrupt change in the closed orbit was correlated to the abrupt change in the horizontal beam size as shown in Fig. 1. This abrupt beam instability caused corresponding abrupt variation in photon intensity for the synchrotron light users. This abrupt beam instability was never observed in low current regime, e.g. for beam current below $130 \mathrm{~mA}$. The onset of this abrupt orbit distortion was neither predictable, nor periodic. The horizontal and vertical orbits shifted by amounts that appeared to be random around the ring. The global orbit feedback system was turned on during this time when orbit shifts took place, and this might have disrupted a natural tendency of orbit shifts with betatron shape. The variation of horizontal beam size is on the order of $30 \mu \mathrm{m}$. There was no abrupt change observed in the vertical beam size. The transverse beam size was measured from the profile of synchrotron light emitted from a bending magnet where the dispersion function is nonzero in the horizontal plane. 


\subsection{Longitudinal Beam Spectrum vs. Horizontal Beam Size}

It was noticed that the unpredictable, abrupt change in the horizontal beam size was correlated to the change in longitudinal beam spectrum and the apparent fill pattern of beam current. Figure 2 depicts the fill pattern corresponding to the case without the abrupt change in horizontal beam size. The longitudinal beam spectrum corresponding to the fill pattern in Fig. 2 is shown in Fig. 3. The rf frequency is $500 \mathrm{MHz}$ and the harmonic number is 200 for SRRC storage ring. A closer view of the unstable mode at $587 \mathrm{MHz}$ revealed a strong longitudinal dipole oscillation as shown in Fig. 4.

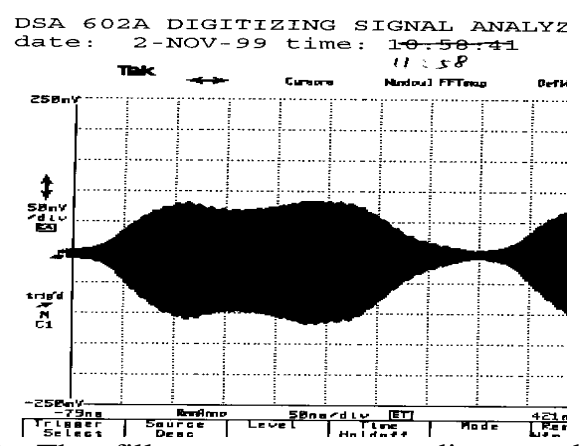

Figure 2: The fill pattern corresponding to the case without the abrupt change in horizontal beam size.

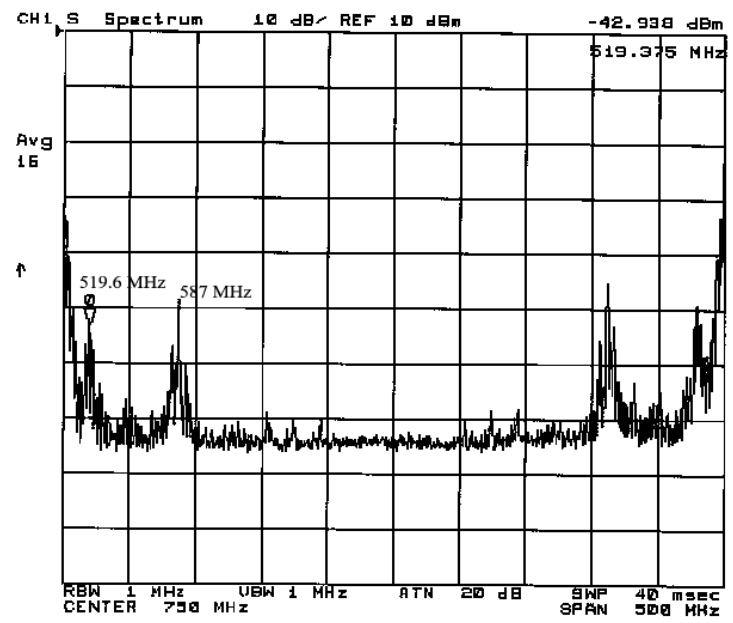

Figure 3: The longitudinal beam spectrum corresponding to the case without the abrupt change in horizontal beam size. Two prominent modes were observed near $520 \mathrm{MHz}$ and $587 \mathrm{MHz}$. The rf voltage modulation was on. The frequency span is from $500 \mathrm{MHz}$ to $1 \mathrm{GHz}$.

When the abrupt change in horizontal beam size occurred, the apparent fill pattern and the longitudinal beam spectrum switched to different patterns as shown in Figs. 5 and 6 respectively. It was realized that the existence of unstable mode at $587 \mathrm{MHz}$ was related to the abrupt change in horizontal beam size and transverse closed orbit.

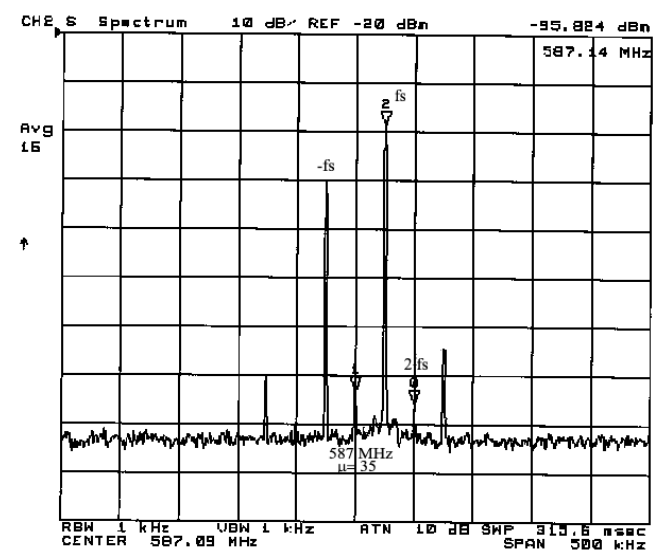

Figure 4: The zoom-in view of unstable mode at $587 \mathrm{MHz}$. The spectrum is centered at the $235^{\text {th }}$ revolution harmonic. The synchrotron frequency is $25 \mathrm{kHz}$. $50 \mathrm{kHz} /$ division.

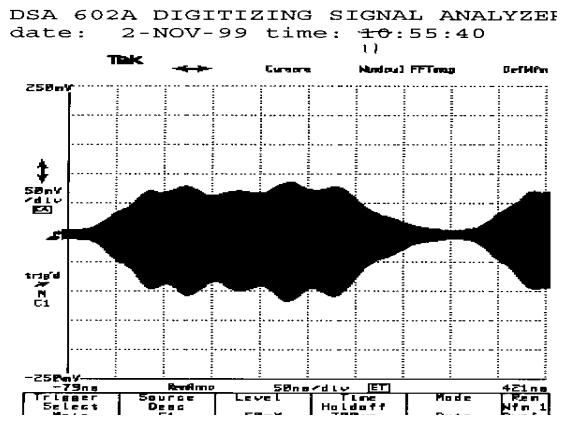

Figure 5: The fill pattern corresponding to the case with the abrupt change in horizontal beam size.

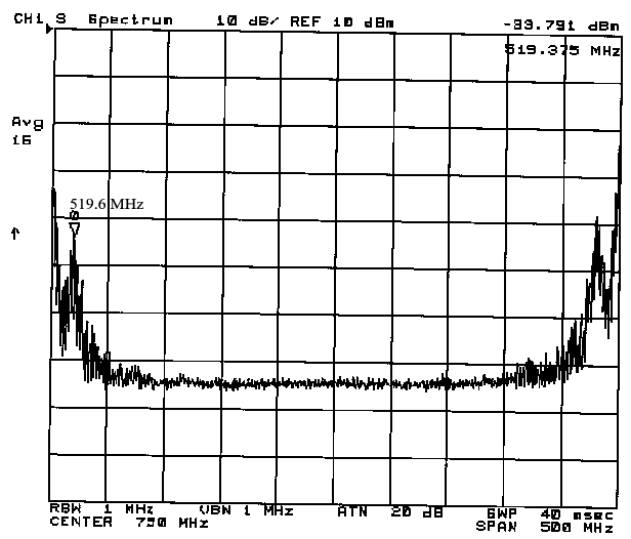

Figure 6: The longitudinal beam spectrum corresponding to the case with the abrupt change in horizontal beam size. The unstable mode near $587 \mathrm{MHz}$ was no longer there. The rf voltage modulation was on. The frequency span is from $500 \mathrm{MHz}$ to $1 \mathrm{GHz}$.

\section{CORRELATION WITH HIGH-ORDER MODES OF RF CAVITIES}

The rf section of storage ring is composed of two Doris-type cavities interconnected by a circular beam pipe with a radius of $7.085 \mathrm{~cm}$. The cutoff of waveguide modes for the circular beam pipe is $1.241 \mathrm{GHz}$ for $\mathrm{TE}_{11}$ and $1.622 \mathrm{GHz}$ for $\mathrm{TM}_{01}$ mode. Above cutoff, there is a crosstalk of HOMs between two rf cavities. 


\subsection{Calculated HOMs of rf cavities}

The layout model of a Doris cavity is shown in Fig. 7. The original design of the Doris cavity was modified. The HOM damping antenna was replaced by a tuning plunger. The longitudinal HOMs for the dual-cavity rf section was calculated by using a 3D rf code GdfidL[3]. Calculated results for a 2D model of rf section are given in Table 1 [4]. Only those modes below the waveguide cutoff were calculated. The growth rate was calculated for the worst case, i.e. on resonance, $200 \mathrm{~mA}$ equally-spaced bunch train. The radiation damping rate in the longitudinal direction is $0.176 \times 10^{3} / \mathrm{sec}$. The disappearance of the unstable mode at $587 \mathrm{MHz}$ in the beam spectrum was related to the abrupt beam instability. This unstable mode corresponds to the longitudinal coupled bunch mode number 37 driven by the HOM at $1590 \mathrm{MHz}$.

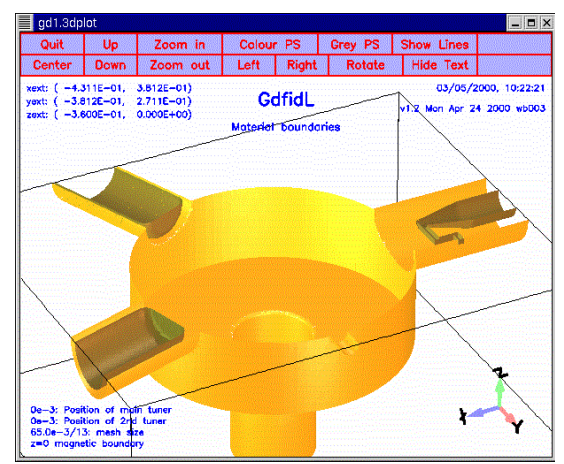

Figure 7: The layout of a Doris cavity in the rf section. The HOM damping antenna was replaced by a tuning plunger.

Table 1: Calculated Longitudinal HOMs of Dual-Cavity RF Section in SRRC Storage Ring

\begin{tabular}{|l|l|l|l|l|}
\hline $\boldsymbol{f}[\mathbf{M H z}]$ & $\begin{array}{c}\mathbf{R} / \mathbf{Q} \\
{[\boldsymbol{\Omega}]}\end{array}$ & Loaded Q & $\begin{array}{c}\boldsymbol{\tau}^{-\mathbf{1}} \\
{\left[\mathbf{1 0}^{\mathbf{3}} / \mathbf{s e c}\right]}\end{array}$ & $\begin{array}{c}\text { Mode } \\
\text { number }\end{array}$ \\
\hline 502.412 & 77.7 & 17100 & 26 & 0 \\
\hline 502.413 & 77.7 & 17100 & 26 & 0 \\
\hline 743.099 & 32.2 & 2243 & 2.2 & 98 \\
\hline 743.101 & 32.2 & 2243 & 2.2 & 98 \\
\hline 1198.896 & 7.4 & 2857 & 1 & 80 \\
\hline 1198.898 & 7.4 & 2857 & 1 & 80 \\
\hline 1292.610 & 5.1 & 3059 & 0.8 & 118 \\
\hline 1292.614 & 5.1 & 3060 & 0.8 & 118 \\
\hline 1590.175 & 4.6 & 3183 & 0.9 & 37 \\
\hline 1590.213 & 9.2 & 3184 & 1.8 & 37 \\
\hline 1701.892 & 3.1 & 3353 & 0.65 & 81 \\
\hline 1702.373 & 1.4 & 3339 & 0.3 & 82 \\
\hline
\end{tabular}

\subsection{Detuning of HOMs by RF Plungers}

We tried to avoid those unstable modes by detuning the frequencies of HOMs. The HOM tuning plunger was moved back and forth in order to find an optimum position while the other plunger was moved to maintain the frequency of accelerating mode. We could not find a setting such that all unstable modes could be avoided. The frequency shift of HOMs vs. position of HOM tuning plunger was simulated with GdfidL and a 3D model of a single cavity. The simulated results are shown in Fig. 8.
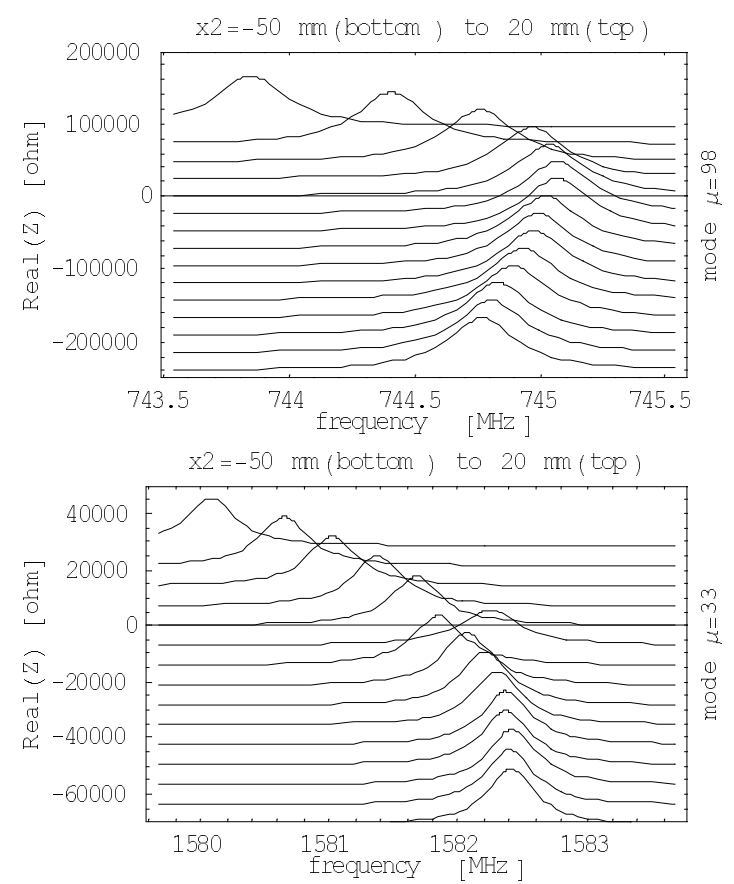

Figure 8: The frequency shift of HOMs vs. position of HOM tuning plunger. The rf plunger was moved at $5 \mathrm{~mm}$ per step. The fundamental rf frequency was held fixed by adjusting the first plunger.

\section{DISCUSSIONS}

The pattern and the scale of frequency shift for each HOM are not the same as shown in Fig. 8. Therefore, it is impossible to find a setting that all HOMs are avoided. Temperature variation has been used as a control, but was not too effective. The final solution in use for the present operation is to avoid the unstable mode driven by HOM at $1590 \mathrm{MHz}$ with tuning plungers and apply the rf voltage modulation to the beam as well. After the HOM detuning, the abrupt change in the horizontal beam size and transverse orbit is no longer observed in user operation. An upgrade by replacing those two Doris cavities with a superconducting rf cavity developed by Cornell University is currently under way[5]. A longitudinal bunch-by-bunch feedback system is also being developed[6].

\section{REFERENCES}

[1] M.H. Wang et al. , IEEE PAC'95 (1995), p.2971.

[2] M.H. Wang et al., IEEE PAC'99 (1999), p.2837.

[3] W. Bruns, IEEE PAC'99 (1999), p.2767.

[4] P.J. Chou, "Summary of Beam Instability Studies for the TLS Storage Ring", SRRC/OP/00-02, SRRC report(2000).

[5] G.H. Luo et al., SRRC Newsletter, No.46 (2000).

[6] W.K. Lau et al., EPAC'00 (2000), p.1918. 